\title{
IoTIZER: A Versatile Mechanical Hijacking Device for Creating Internet of Old Things
}

\author{
Hyungjun Cho' \\ lolo660@kaist.ac.kr
}

\author{
Han-Jong Kim ${ }^{2}$ \\ hanjongkim@kpu.ac.kr
}

\author{
JiYeon Lee ${ }^{1}$ \\ ji.lee@kaist.ac.kr
}

\author{
Chang-Min Kim ${ }^{1}$ \\ peterkim12@kaist.ac.kr
}

Jinseong Bae ${ }^{1}$
bjsjs3002@kaist.ac.kr

Tek-Jin Nam ${ }^{1}$

tjnam@kaist.ac.kr

Department of Industrial Design, KAIST, Daejeon, Korea, Republic of ${ }^{1}$

Department of Design Engineering, Korea Polytechnic University, Siheung-si, Gyeonggi-do, Korea, Republic of ${ }^{2}$

\section{ABSTRACT}

Mechanical hijacking of physical interfaces is a costeffective method for providing an Internet of Things (IoT) experience. However, existing mechanical hijacking devices (MHD) have limited applicability and usability. This pictorial introduces a research through design project on IoTIZER, which is easy to use and versatile MHD. We report on a design process from identifying a design space, conducting an iterative design and prototyping. We present the final design's hardware, software, usage scenario and implementation details. We also share lessons from a user study with eight households. The potential value of IoTIZER were appreciated as a versatile MHD. We discuss improvement areas of IoTIZER and implications for creating an IoT environment while preserving the existing conventions.

\section{Authors Keywords}

Internet of Things (IoT); Internet of Old Things (IooT); Mechanical Hijacking; Product Augmentation; Technology Probes; Research through Design.

Permission to make digital or hard copies of all or part of this work for personal or classroom use is granted without fee provided that copies are not made or distributed for profit or commercial advantage and that copies bear this notice and the full citation on the first page. Copyrights for components of this work owned by others than ACM must be honored. Abstracting with credit is permitted. To copy otherwise, or republish, to post on servers or to redistribute to lists, require prior specificpermission and/or a fee. Request permissions from Permissions@ acm.org.

DIS '21, June 28-July 2, 2021, Virtual Event, USA

(C) 21 Association for Computing Machinery.

https://doi.org/10.1145/3461778.3461996

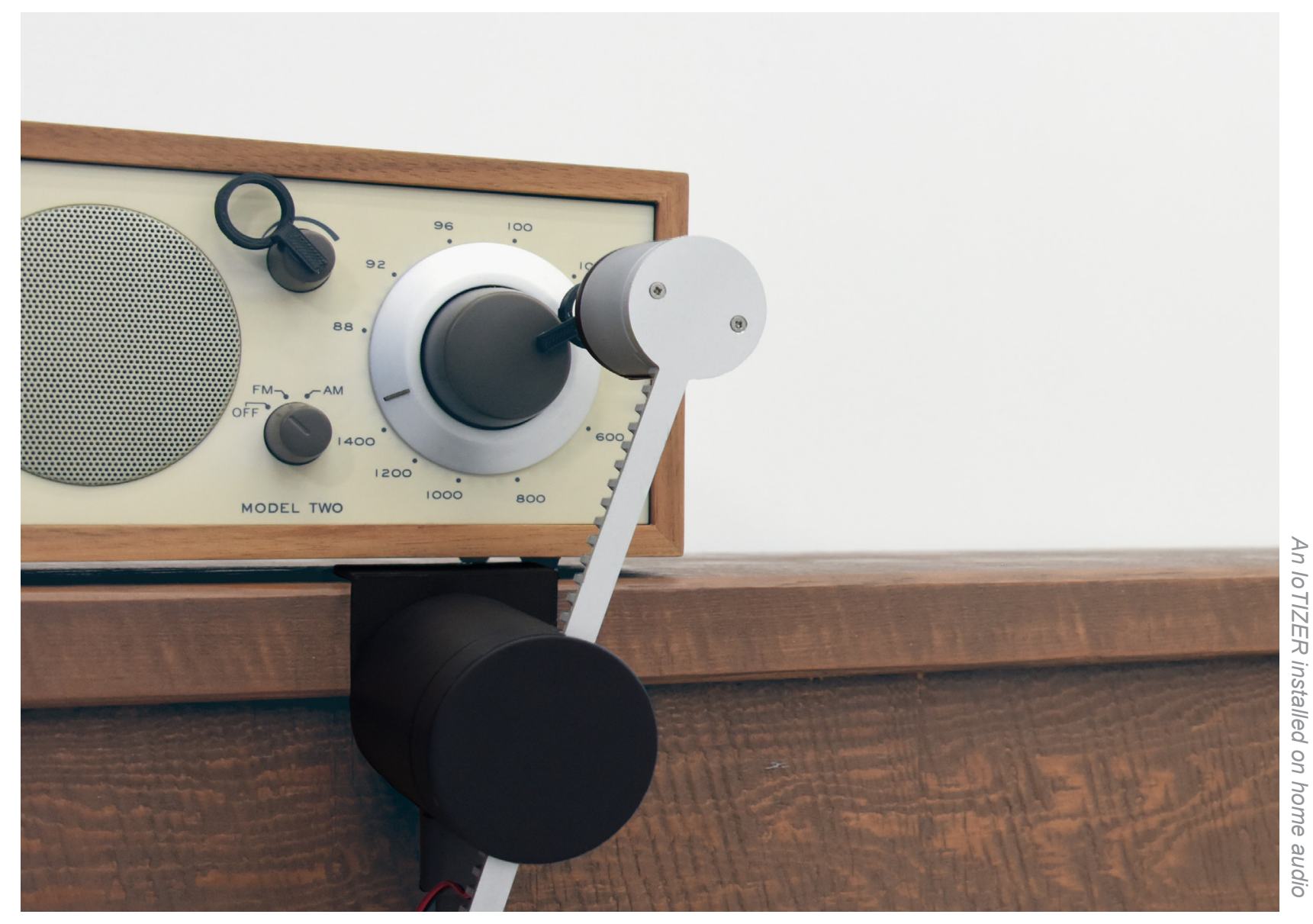




\section{INTRODUCTION}

Although the Internet of Things (IoT) provides great advantages in terms of convenience and personalization [32], it is still challenging for the general public to adopt IoT technology in everyday life. One problem is the cost and time demanded [5] to replace existing products with IoT products. As an alternative, several researchers presented do-it-yourself (DIY) methods [3, 30], and kits for quick and easy IoT development have been introduced [21, 29]. Such methods and kits can help implement the technical features of IoT, such as wireless connection and remote accessibility, common users still have difficulty practically applying these features in real life due to low robustness, low security, and technical difficulties.

To mitigate the problems, ideas for enabling the IoT experience with existing products have been proposed. We call this augmented product, the Internet of Old Things (IooT). To create an IooT, Davidoff et al. proposed mechanical hijacking using actuators to replace human manipulation of the physical interface [10]. Because of their cost-effectiveness and low barriers, several mechanical hijacking devices (MHD) were introduced in the IoT market $[18,35]$. However, existing MHD are not versatile enough for use in domestic products, so the user's experience is limited.

In this pictorial, we introduce a research through design project on IoTIZER, a versatile MHD for common users that creates IooT. The final IoTIZER design was completed after an investigation on the design space of MHD in a domestic environment and a series of design judgements and prototyping. We share the learnings in the design process and the design rationales for the features of the final design. We also report on a technology probe study [16] to understand how users perceive an IoTIZER and what they expect from it. Based on the design iteration and the field study, we discuss the opportunities and challenges of an augmentation approach that uses MHD to add IoT functions while maintaining existing products.

\section{RELATED WORKS}

\section{Supporting End Users' loT Experience}

As IoT affects individuals' lives, various researches have been conducted to understand end-users' experiences in the domestic IoT environment $[2,4,8,23,36]$. Especially the diversity of domestic environments and individual needs among users [24, 34] has resulted in much research on end-user development [9], particularly in smart home contexts. For example, various researchers have proposed trigger-action programming $[13,25$, 26]. Despite some weaknesses, commercial systems including IFTTT [17] use such methods and encourage the current DIY smart home culture. However, finding the IoT products needed for smart homes is still challenging. Not only is it technically difficult to install and use [1], but fundamentally, the IoT products supplied by manufacturers is limited to lights, smart speakers, and a few white goods.

We expected that the use of IooT, which adds IoT functions to existing products, could solve this problem. We explored how MHD can help successfully create an IooT by designing exemplary cases of versatile MHD

\section{Replacing Human Control for loT Experience}

After Davidoff et al. proposed a notion of mechanical hijacking to enable computational control by hijacking a product's physical interface [6], various types of MHD were introduced. Switcher [18] and LiftMaster [33] can be mounted over wall switches and door locks, respectively. They are easy to install and use but are designed only for target products. Switchbot [35] and Microbot-push [25] are slightly more versatile but still limited to a single push-type interface. Although Smartians [12] can create motions other than pushing via modular header parts, the 'one device for one interface' approach is still difficult to use for products with diverse controls.

As the aforementioned designs limit users' opportunities, several researchers have proposed design tools for creating customized MHD. Retrofab [28] generates a set of new physical interfaces that can be remotely controlled via 3D scanning and software tool. Robiot [19] automates the operation mechanism of everyday objects via a user demonstration video. However, these systems require advanced digital fabrication and physical computing skills. Thus we introduce the design of an easy-to-use and versatile MHD.

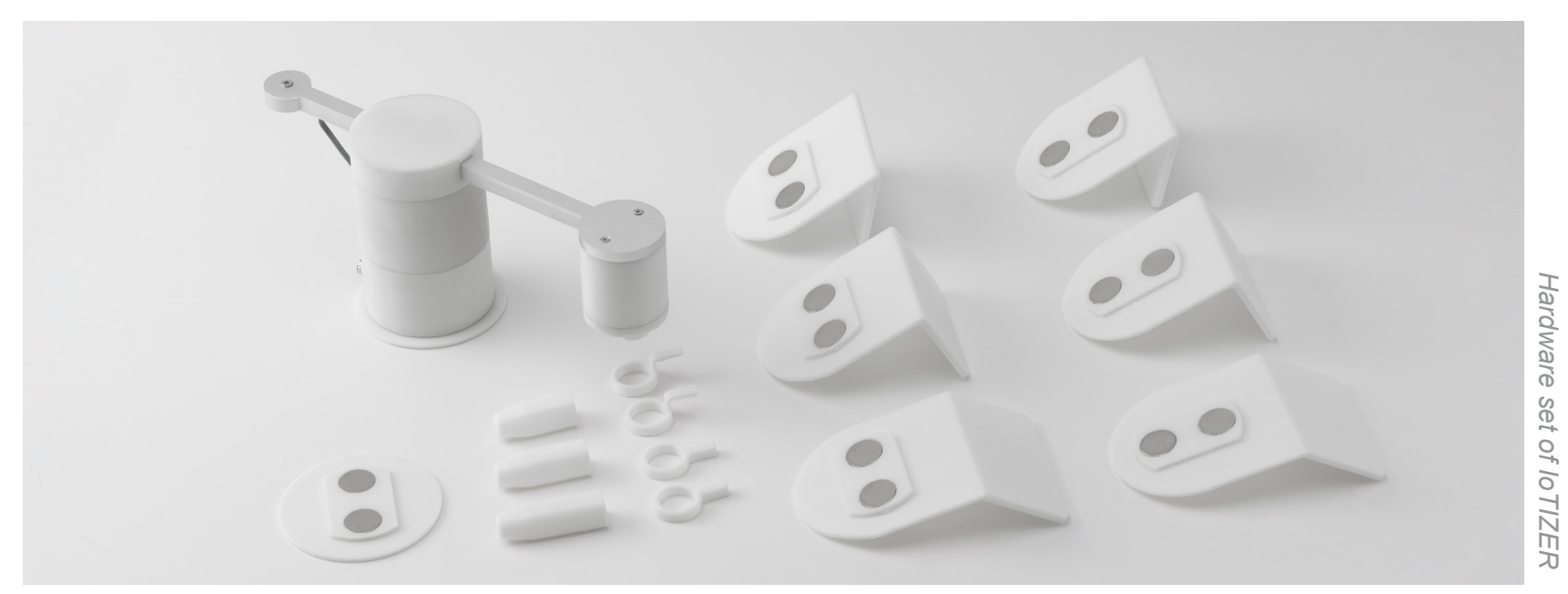




\section{DESIGN SPACE OF MHD FOR DOMESTIC ENVIRONMENT}

We explored a design space to understand the degree of versatility of MHD in a domestic environment. We developed it to incorporate a wide range of everyday products and to focus on manipulation via MHD instead of humans' direct control [6]. The design space is applicable to identify the requirements and design elements of MHD for various everyday products.

We inspected the shapes and layouts of the physical interfaces of various products in a domestic environment. We collected 723 photos of products from 19 households. We recruited one member from each household and asked them to photograph all the products including physical interfaces in their homes. We then conducted the iterative card sorting process with labeled photos.

We identified three aspects to consider for MHD design. The first is the motion path when manipulating the interface. Most physical interfaces could be manipulated by pushing, rotating, or dragging. To fully utilize the product's functionality, however, detailed operations (e.g. long-pressing a button, turning a knob at a certain angle) were necessary. The second aspect is the form of the interface. Some products had only one interface component, but many products had multiple and even complex interfaces. The third aspect is the product's movability. Domestic products included a built-in type embedded in a wall or cabinet, an installed type that is difficult to move due to surrounding facilities or heavyweight, a placed type that has its position but is not fixed, and an unlocated type with a position that changes frequently.

Figure 1 shows a design space with movability as the horizontal axis and the form of the interface as the vertical axis. The control type of each interface is colorcoded. It also visualizes the distribution of products in the domestic environment regarding the three aspects.

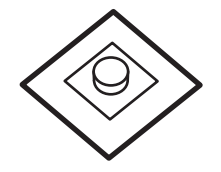

Built-in

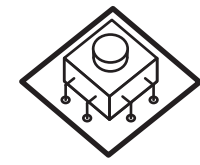

Installed

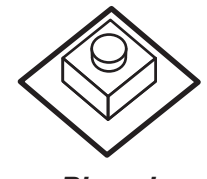

Placed

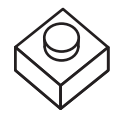

Unlocated
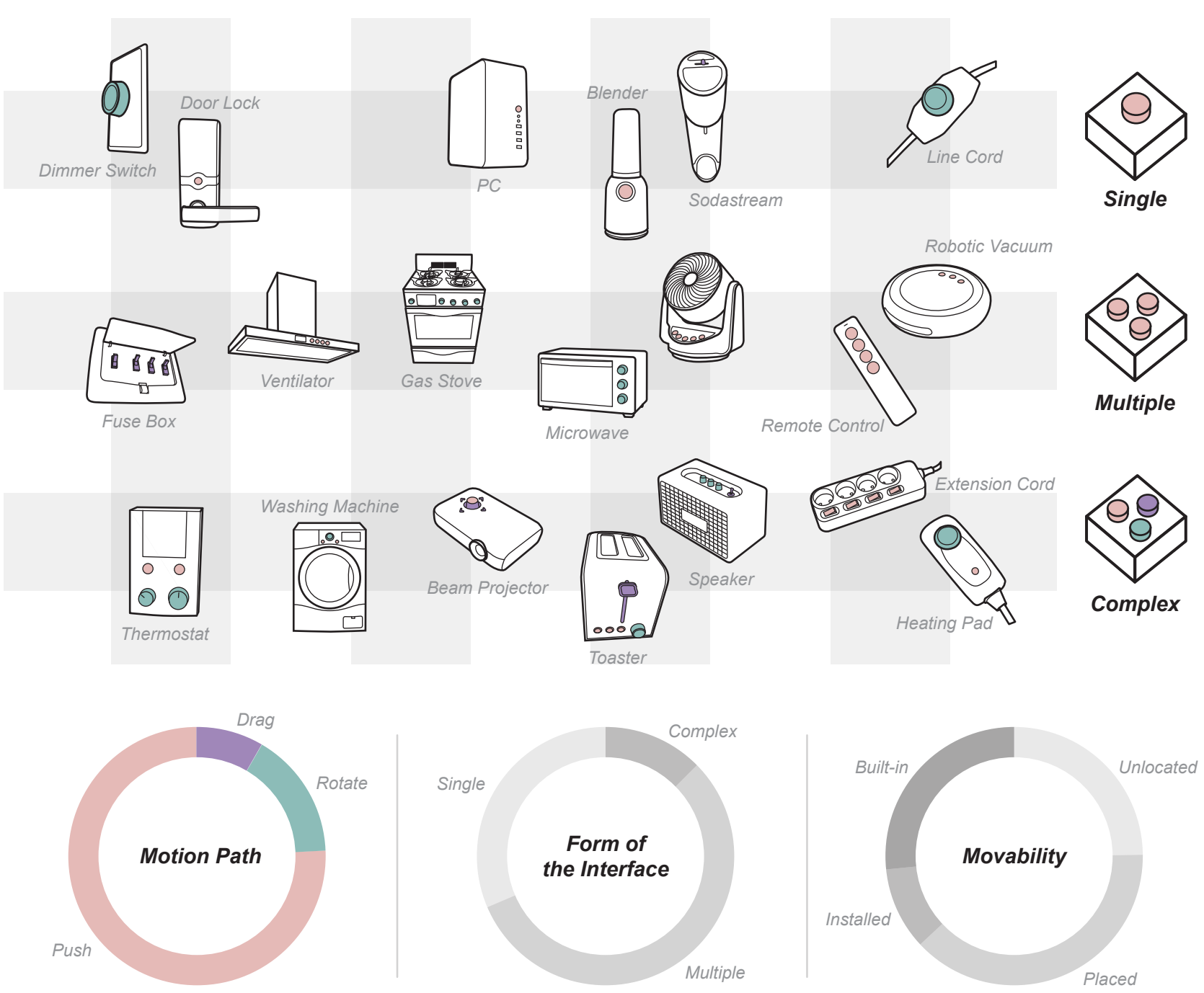


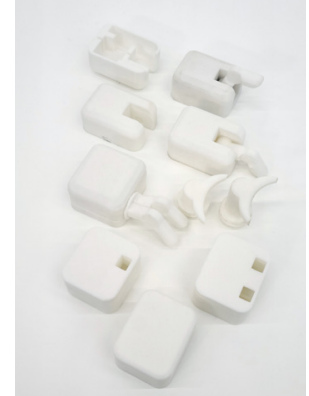

Boxed module

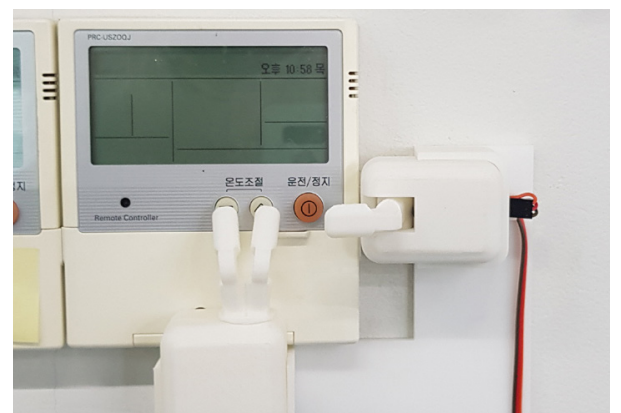

Testing on control panel

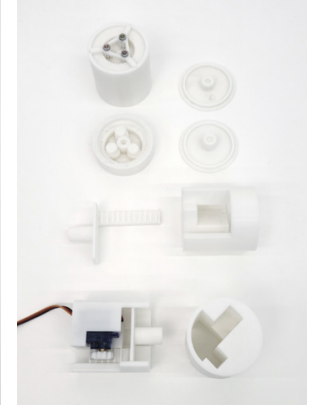

Cylindrical module

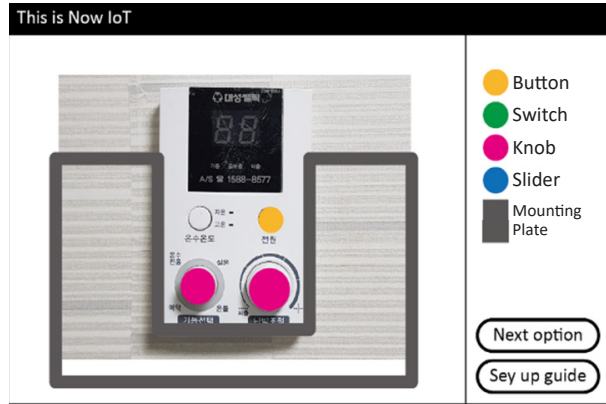

Initial plan of design too

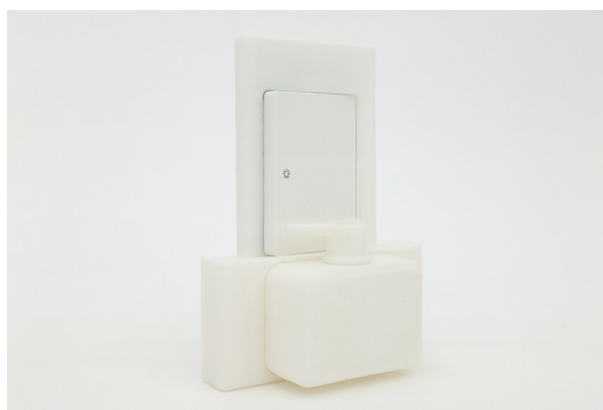

Testing on wall switch

\section{FIRST PHASE OF DESIGN EXPLORATION:}

ACTUATING MODULES, CUSTOM MOUNTING PLATE, AND DESIGN TOOL

We first planned to design tiny modules for each interface type and to assemble them with custom mounting plates. We also considered a design tool that allowed users to easily design a mounting plate according to a target product's shape and interface layout. We expected this method could effectively adapt to diverse domestic products. Through iterative prototyping, we explored the actuating modules of various shapes and mechanisms, while testing mounting plates for switches and air conditioner controllers

However, a limitation to miniaturizing the actuating module, made it difficult to create a mounting plate for an interface with a dense layout or located in the middle of the product. Also, such an idea does not resolve the existing issues of the DIY approach. It would have low robustness, and developing aesthetic aspects is difficult compared to commercial products. Moreover, it is challenging to use for people who lack digital fabrication knowledge and experience.

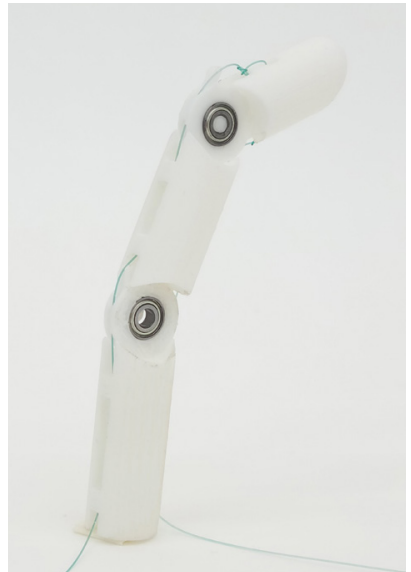

Tendon-based
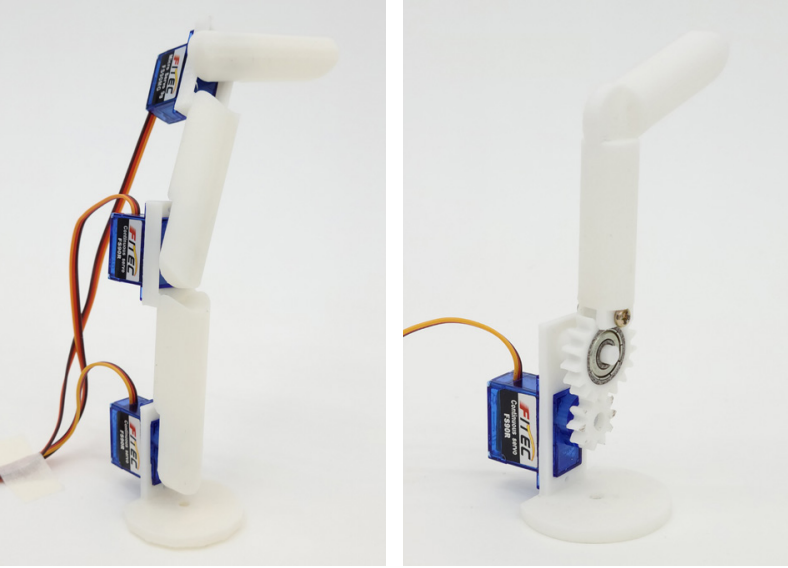

One motor for one joint

Bottom joint only

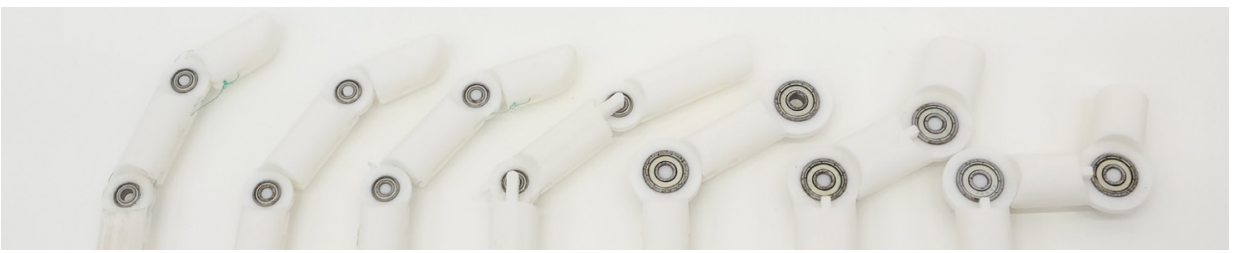

Number of prototypes

\section{SECOND PHASE OF DESIGN EXPLORATION: REMOTE FINGER}

Since we identified the necessity of 'one-for-all-device' that can manipulate multiple interfaces, we examined a finger-shaped device that mimics humans. We expected the device's shape to provide an affordance for functionality and a playful experience, as well as a finger-like joint structure that allows us to implement various movements. We tried three mechanisms through iterative prototyping. First, we used a single motor and string for tendon-based control $[31,37]$. Second, we used the motor for each joint [22]. And third, we fixed the shape of the finger and installed the motor only at a lower joint.

However, the finger-shaped device could not generate enough force to manipulate the interface. The joint structure enables flexible motions but includes many disadvantages in transmitting force, such as the distance from the motor to interface and elasticity at the joint. Besides, the elongated shape gave a visually unpleasant feeling when installed. 

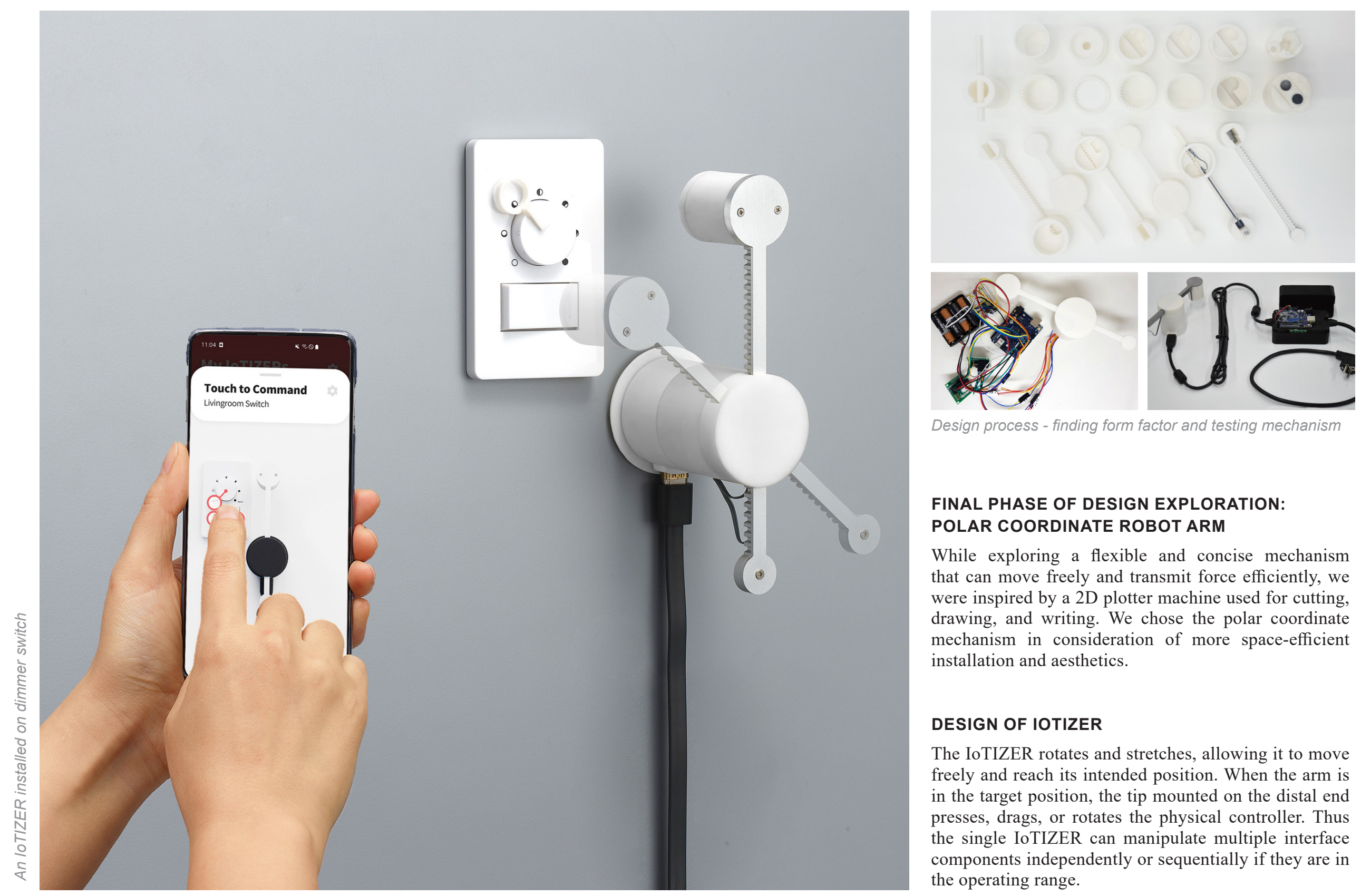

Design process - finding form factor and testing mechanism

\section{FINAL PHASE OF DESIGN EXPLORATION:}

POLAR COORDINATE ROBOT ARM

While exploring a flexible and concise mechanism that can move freely and transmit force efficiently, we were inspired by a 2D plotter machine used for cutting, drawing, and writing. We chose the polar coordinate mechanism in consideration of more space-efficient installation and aesthetics.

\section{DESIGN OF IOTIZER}

The IoTIZER rotates and stretches, allowing it to move freely and reach its intended position. When the arm is in the target position, the tip mounted on the distal end presses, drags, or rotates the physical controller. Thus the single IoTIZER can manipulate multiple interface components independently or sequentially if they are in the operating range. 
COMPATIBLE WITH VARIOUS EXISTING PRODUCTS The modular end parts allow IoTIZER to be installed on products with various shapes. The holder, which is attached to the target product and fix the IoTIZER, has several variations to fit a target product. When the pre-designed holder is unavailable, users can design a custom holder. The tip has three lengths to choose from based on the vertical distance to the interface.
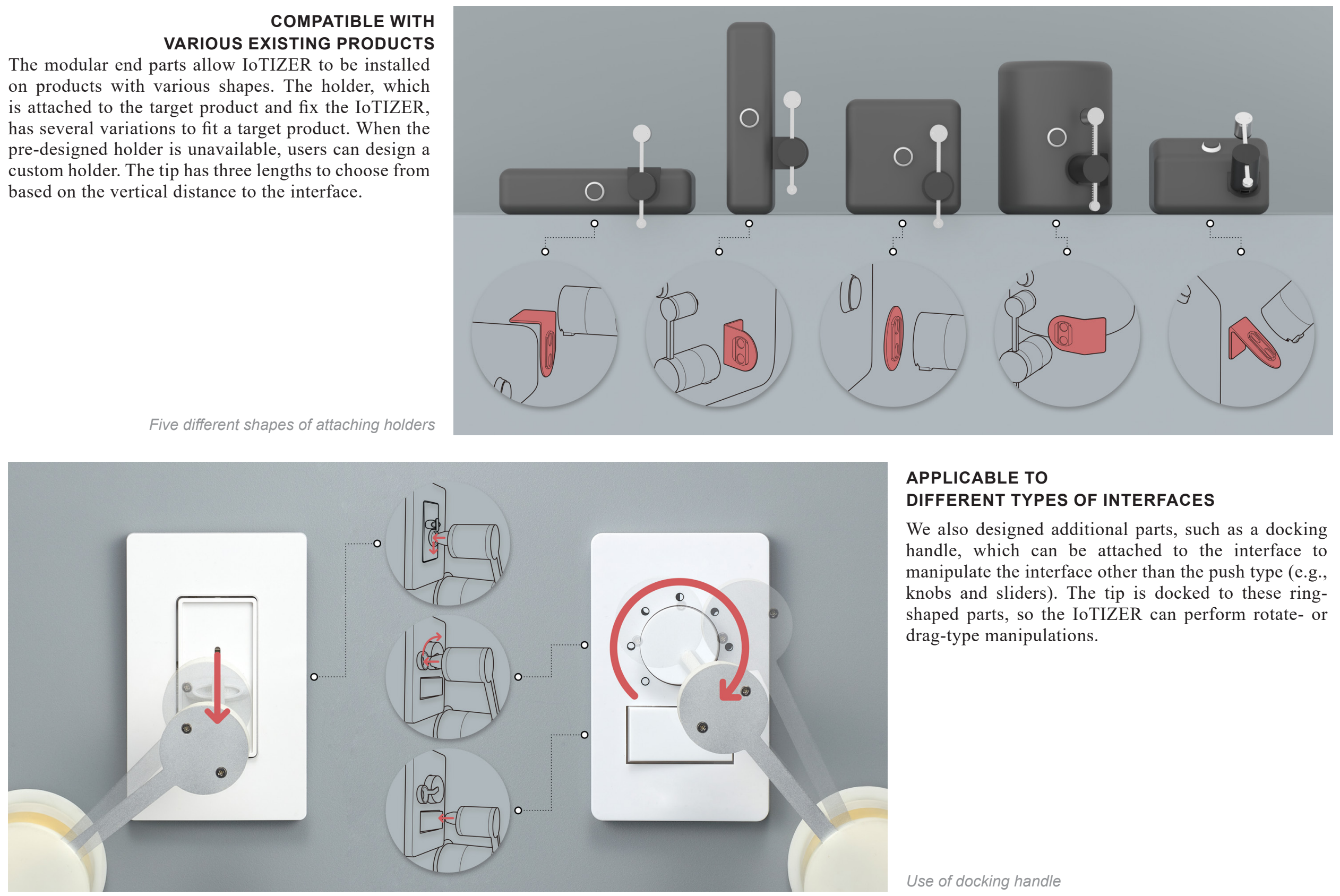

\section{APPLICABLE TO \\ DIFFERENT TYPES OF INTERFACES}

We also designed additional parts, such as a docking handle, which can be attached to the interface to manipulate the interface other than the push type (e.g., knobs and sliders). The tip is docked to these ringshaped parts, so the IoTIZER can perform rotate- or drag-type manipulations. 


\section{SOFTWARE APPLICATION}

The associated software of IoTIZER was developed as a web-based application, so users can manage and control multiple IoTIZERs from any platform. Users can protect data and personalize content through a social login (Figure 2a). To provide an intuitive experience, the IoTIZER app utilizes photo-based interactions. We referred to previous research on human-robot interaction related to using 2D images to generate the desired movement in robots $[11,20]$. It simplifies the process of registering a new device by taking a photo (Figure $2 b$ ). Because the created photo is displayed in a gallery on the device and action list (Figure 2c, 2f), it is easy to manage multiple items. Users can create a familiar control panel by creating GUI control points on the physical interface in the photo (Figure 2d). Photos and control points also create shortcuts for desired sequential manipulation as an action (Figure 2g). By using real-world photos in the interface, existing experience and knowledge of the product can be integrated into the interaction in the app. Also, users can configure the preset action to operate according to desired rules (Figure $2 \mathrm{~h}$ ). For example, it can activate at the desired time or be triggered by voice command.

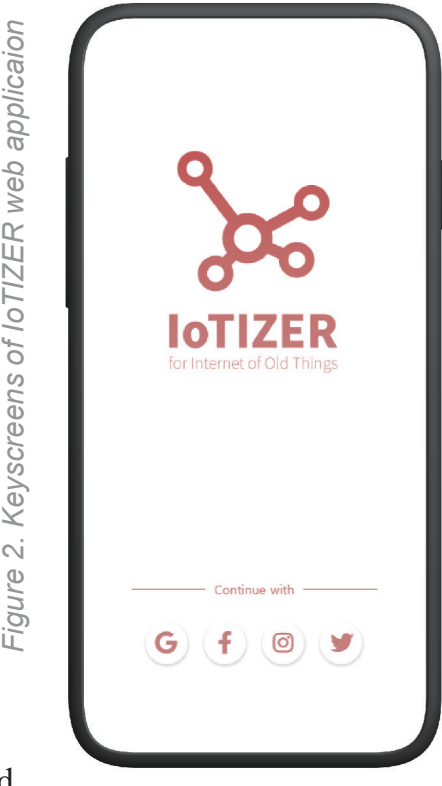

a. Social Login

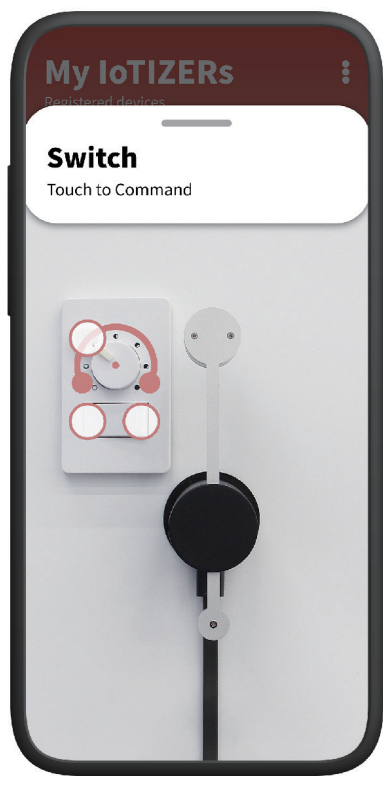

e. Remotely Controlling

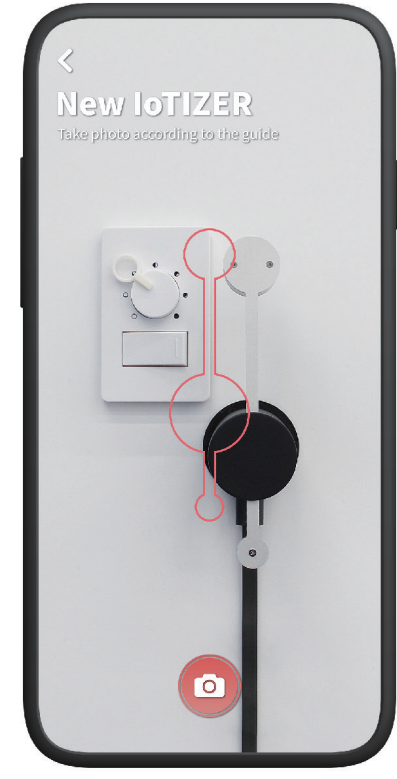

b. Registering New IOTIZER

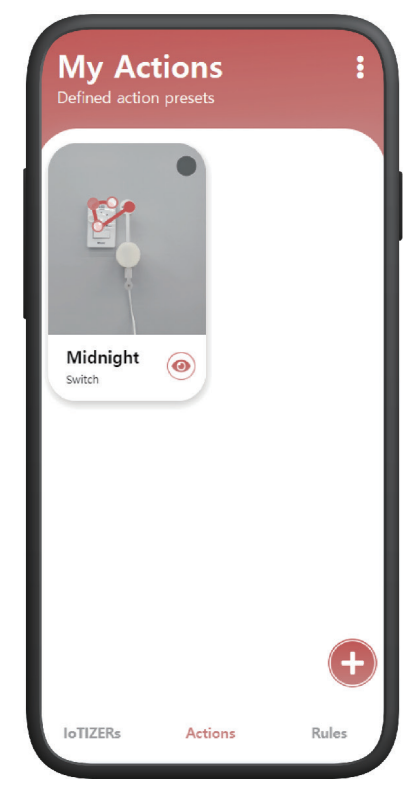

f. Action List

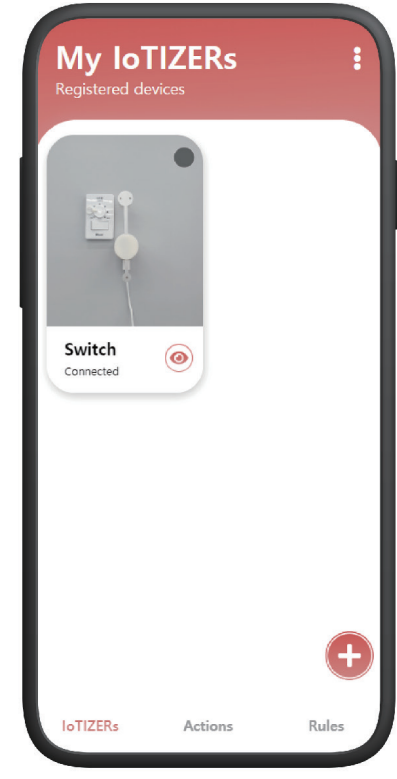

c. Device List

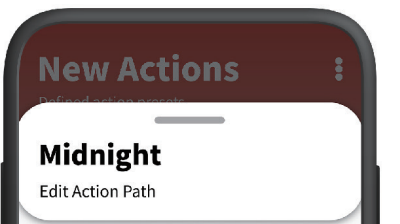

Edit Action Pat

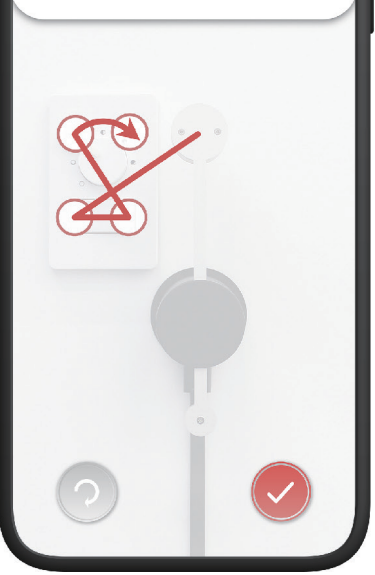

g. Defining New Action

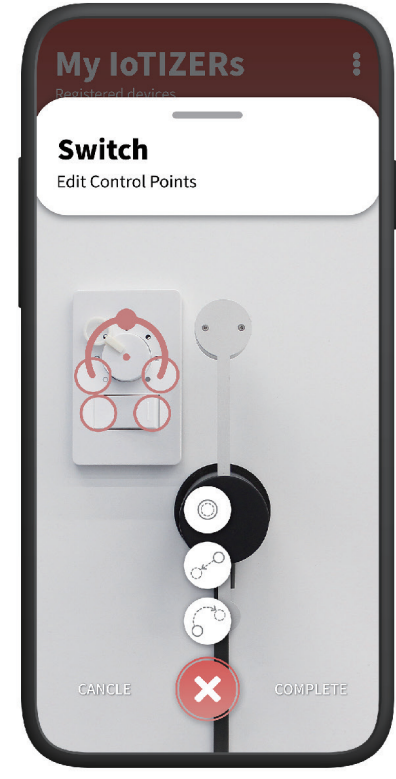

d. Creating Control Porints

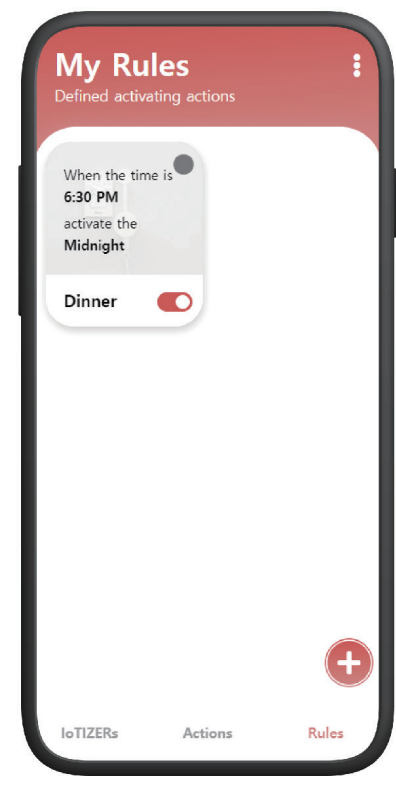

h. Rule List 


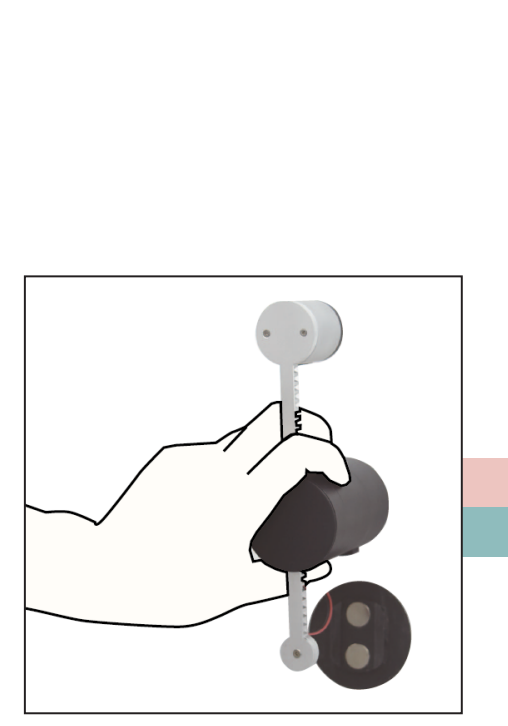

1. Installation

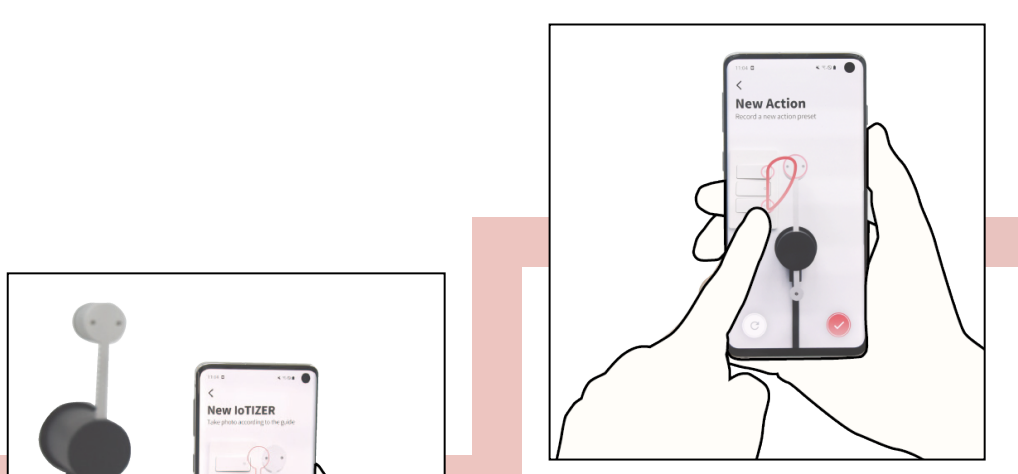

3A. Defining Action

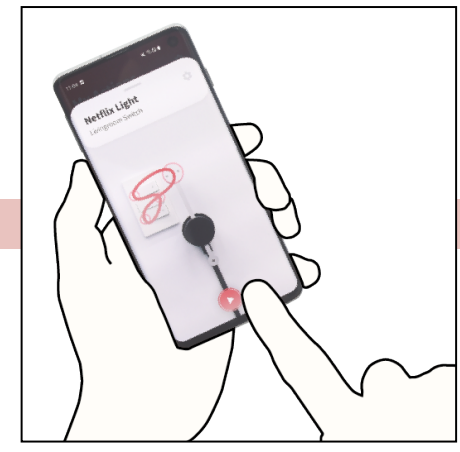

4. Executing Action

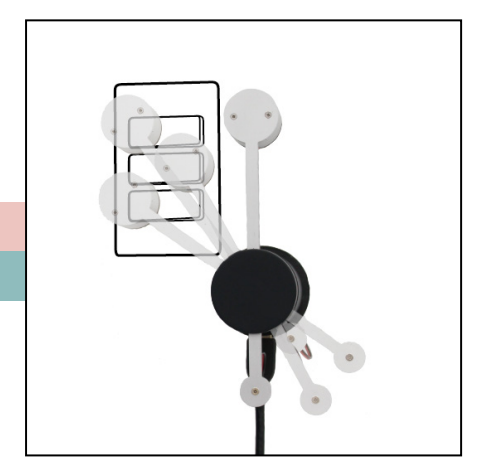

5. IOTIZER Activated

2. Registration

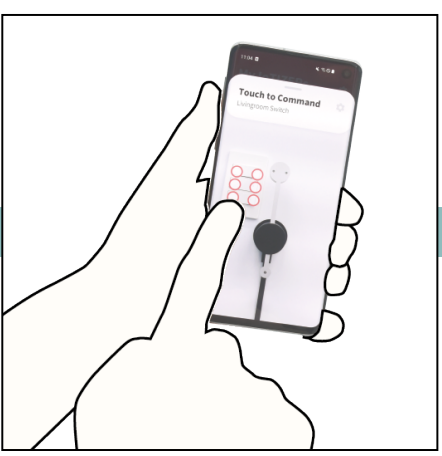

3B. Executing Direct Contro
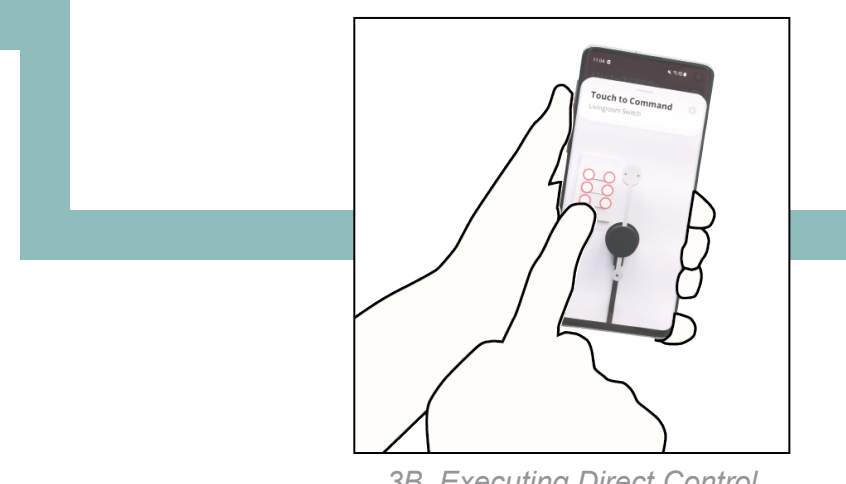

\section{HOW TO INSTALL AND USE}

\section{Installation}

A user selects the suitable shape holder and attaches it to the target product. The user can decide how to position the holder by examining the interface that can be reached. After attaching the holder, the user docks the main body to the holder (Figure 3-1). To register the installed IoTIZER device, the user launches the mobile application and touches the add button on the IoTIZERs tab. The user then takes a photo by locating the device's top view in the visual guide on the screen (Figure 3-2). The user can complete the registration by entering the device name and ID of the interface module (e.g. power switch) and confirming the creation of a new item in the IoTIZERs tab.
Defining control points and direct control

Before using the remote control with IoTIZER, the user must identify and specify the control points. To prevent malfunction and to give precise commands in a mobile environment, the user has to conduct tests to find the coordinates by which IoTIZER can successfully manipulate the interface. Users can open the edit window to create, test, and move three types of control points (i.e. push, drag, and rotate). When the user selects an item, the specified control points appear on the photo used for registration. The user can command the desired manipulation by touching or dragging the control points (Figure 3-3B). The IoTIZER then physically manipulate the interface.
Defining and executing action

Users can create and utilize shortcuts for sequential and complex manipulations in the Actions tab. To define a customized action, the user touches the 'add' button on the Actions tab and draws the path by touching and dragging the control points in the desired order (Figure 3-3A). Then, the application shows the new action item with a visualized path on the photo. When the user touches an item in the action list (Figure 3-4), the IoTIZER sequentially moves to and manipulates the designated points. When the action is completed, the device returns to its default position. The created action can be automatically executed according to conditions set in the rule tab. 


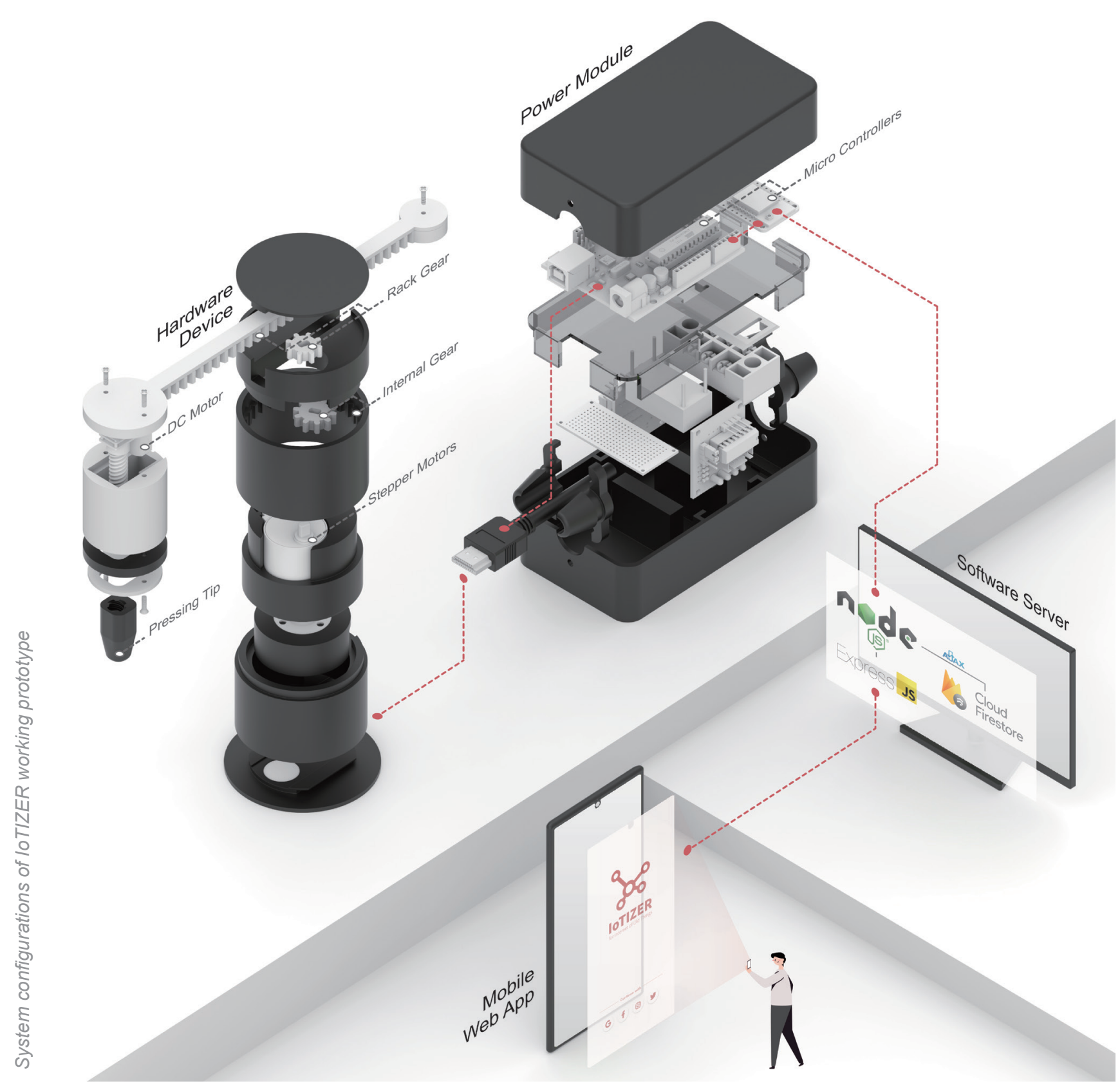

\section{MPLEMENTATION}

\section{Hardware}

The working prototype of IoTIZER (D: $5.5 \mathrm{~cm}, \mathrm{H}: 8.4 \mathrm{~cm}$ ) uses two $5 \mathrm{~V}$ bipolar stepper motors and one geared DC motor. The lower stepper motor rotates the internal parts and bar structure, by meshing with the grounded shell structure's inner gear. Another stepper motor extends the length of the bar (which length is $20.5 \mathrm{~cm}$ ), by generating a linear motion with a pinion gear (the reach is $4 \mathrm{~cm} \sim 13 \mathrm{~cm}$ from the center of the cylinder). The geared DC motor is installed at the end of the bar and is equipped with a screw shaft. The shaft and rectangular hole include a screw-drive mechanism to move the tip up and down. Three motors connect to the power module via a connector, and a slip ring prevents the wires from twisting by rotation. The power module includes an $\mathrm{AC}-\mathrm{DC}$ converter that directly connects to $\mathrm{AC}$ outlets in domestic environments. It also includes an Adafruit Feather HUZZAH with ESP8266 to communicate with the server and Arduino Uno for controlling motors.

\section{Software}

A software server was implemented using the Node. js framework and Express package. The Web interface is rendered on the smartphone screen through the ejs view engine. Users' data stored in Firebase Firestore are transmitted via ajax communication. It uses an HTTPS connection to get permission for the smartphone camera. The server simultaneously connects to the Web application and the Feather ESP8266 board via Socket. io. Created information and modified in the mobile application (e.g., device UID, device and action names, and control points) is updated in Firestore. Each device is identified by a unique ID from the Feather ESP8266 boards. When a user sends a command from the mobile application, the server instantly sends the appropriate command to the target device. The position information is changed from Cartesian to polar coordinates that use a step of the stepper motor as the basic unit. When the Feather ESP8266 board receives data, it transfers the data to the Arduino Uno using I2C communication. 


\section{FEEDBACK FROM USERS}

We collected feedback from target users via a technology probe study comprising co-creation and in-field semi-structured interviews. The purpose of this study was twofold. The first was to verify if IoTIZER is accepted by users as the exemplary of a versatile MHD. Specifically, we focused on the feasibility and usability of IoTIZER in terms of installation and operation in a real domestic environment. We also wanted to understand the opportunities and challenges of IooT creation via MHD. Thus, we used the IoTIZER not only as a prototype but also as technology probes for facilitating an IooT. We expected (1) to introduce MHD ideas that can be applied to various products through the IoTIZER, (2) to promote participants' understanding of IoTIZER's working mechanisms and key functions, and (3) to encourage participants to imagine and immerse themselves in the use of the IoTIZER.

We recruited eight households through the local online community. The 14 participants were diverse in terms of age and their knowledge and experience regarding the IoT. In the feedback sessions, we prepared three items.

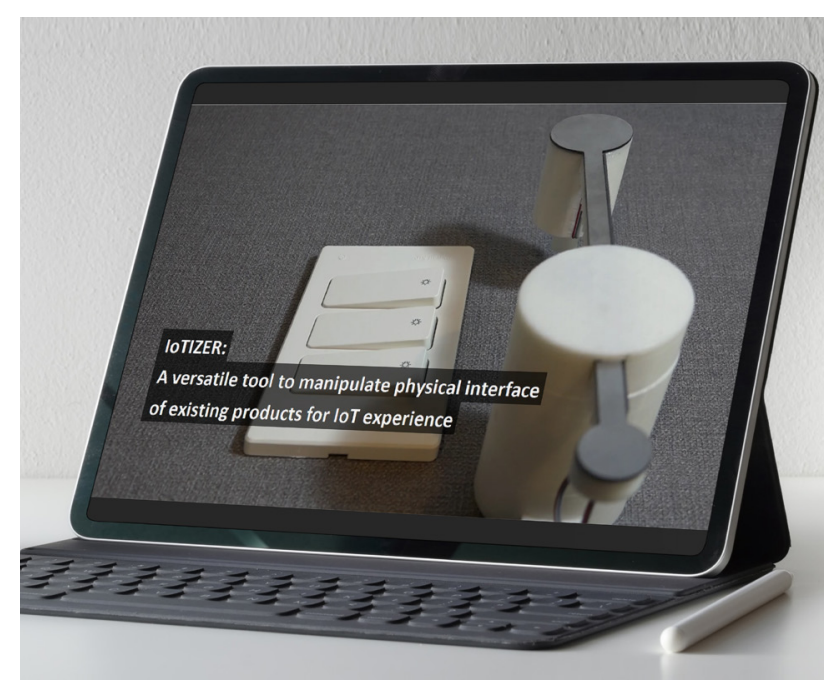

Introductory Video
The first was a 3-minute video introducing the IoTIZER. The second item was a working prototype. An example product for use with the IoTIZER was a switch installed on a temporary wall. The last item was the IoTIZER's mockup kits for installation on various products. The kit comprised three IoTIZER mockups of different sizes, three tip length variations for each size, a holder with seven shapes, several docking handles with two shapes each.

We first introduced the IoTIZER through videos and working prototypes. Then, we ran two or three co-created IooT cases using products in users' homes and the IoTIZER kit. We encouraged users to select the product that reflected their individual needs. Participants could experience the installation and initial setup process. Actions and rules were defined while assuming actual use. In-depth interviews were conducted after the co-creation session. Approximately 265 minutes of interviews were audio-recorded and transcribed. The data was analyzed through iterative analytic induction.

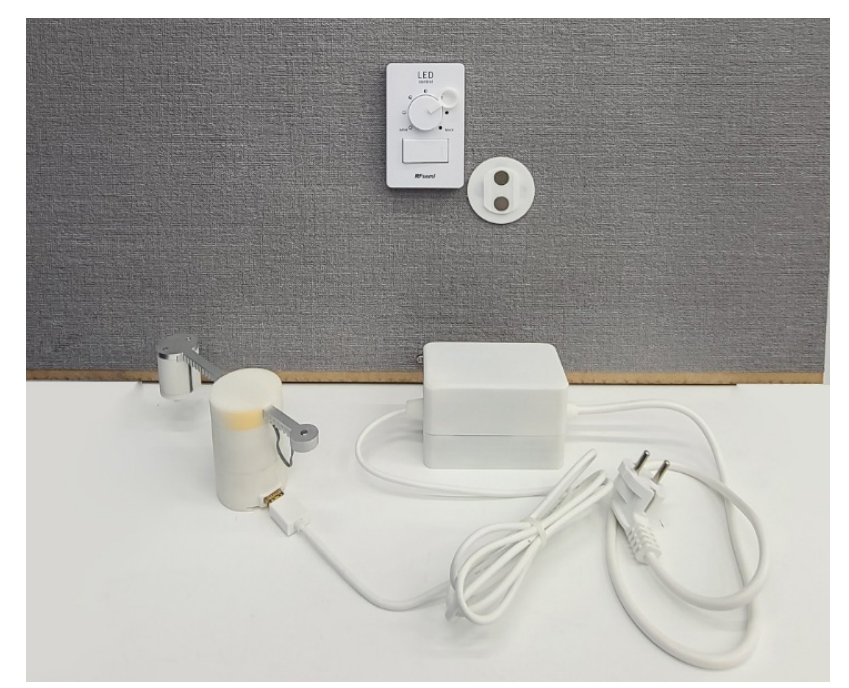

Working Prototype

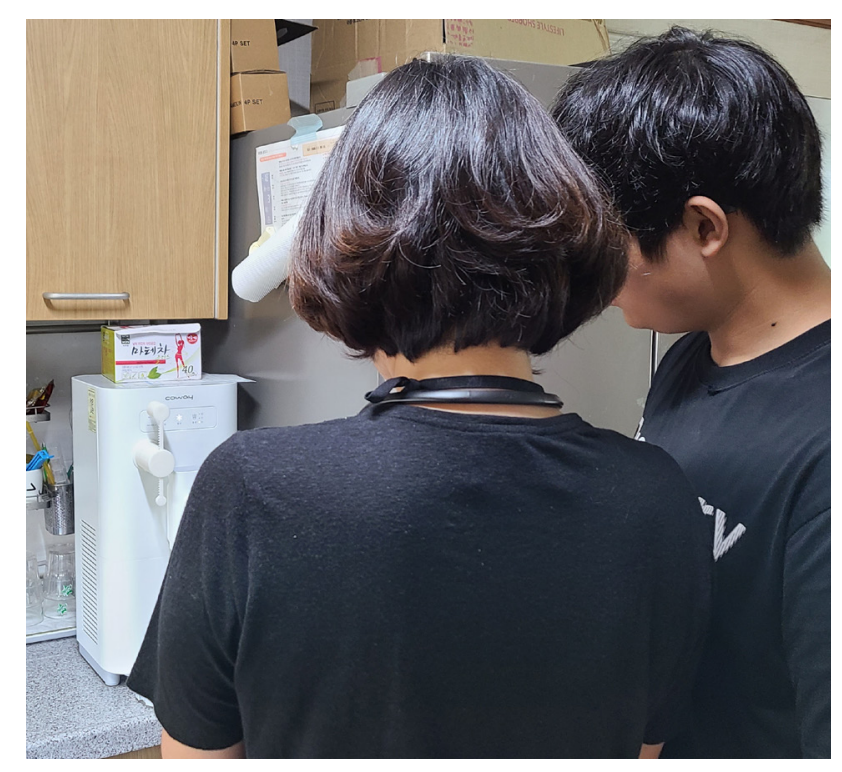

Co-creation session using probe kit in domestic environment $(\mathrm{H} 3)$

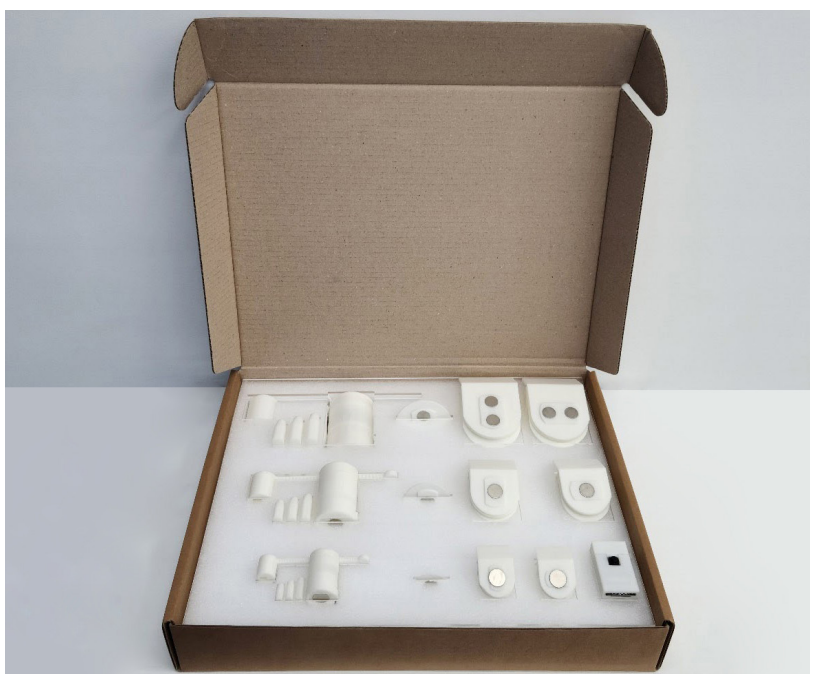

Mockup Kit 


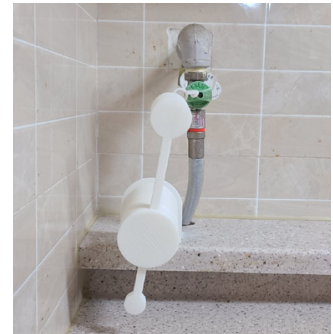

[H1] Gas valve that locks when the user leaves home

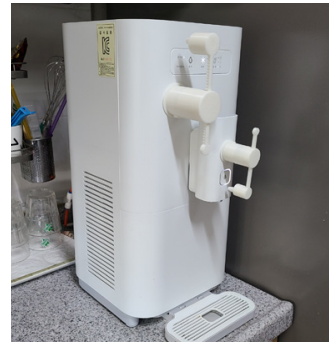

[H3] Water dispenser to help housework intelligently

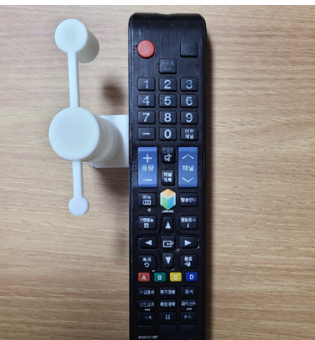

[H5] TV remote control that remembers family member's

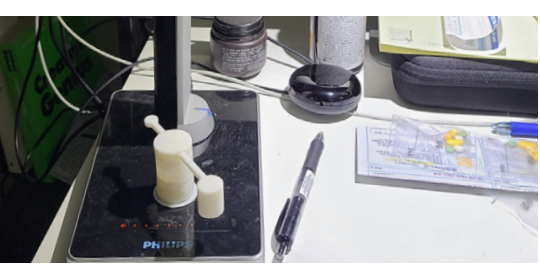

[H8] Desk lamp that remembers the user's preferred brightness

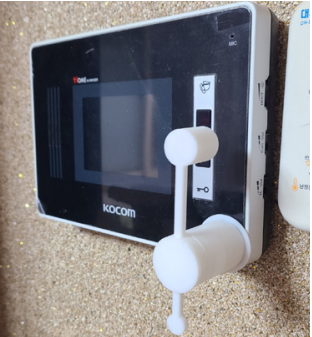

[H1] Intercom controlled by voice command

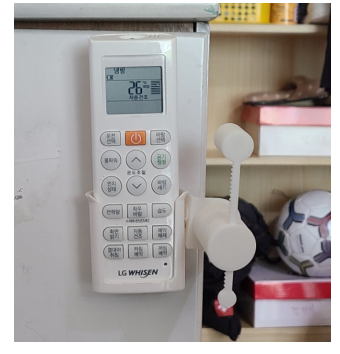

[H3] AC remote control accessible from outside the hom

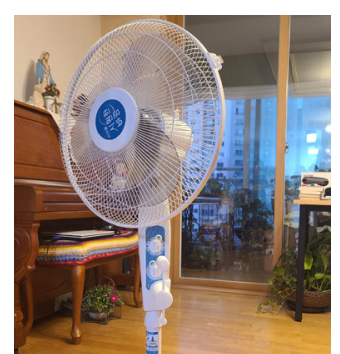
with a mobile phone
[H6] Fan that can be operated

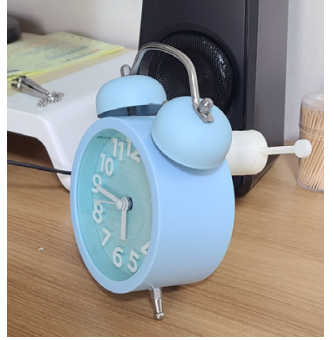

[H2] Analog alarm clock that can be set from mobile phone

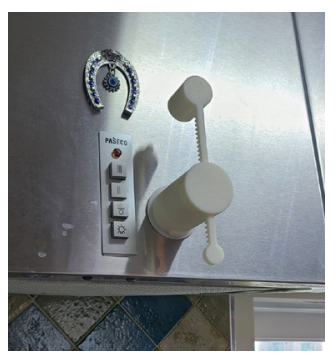

[H4] Ventilator controlled by voice command

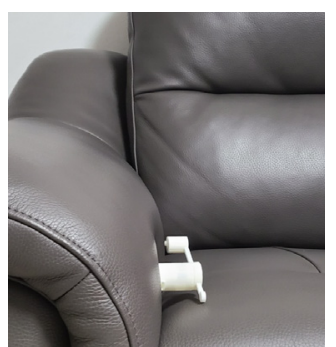
remembers the user's

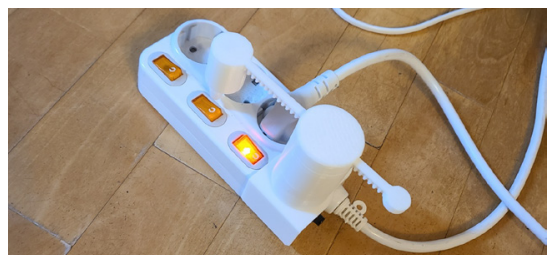

[H6] Extension cord that automatically cuts off the power of unused products
[H7] Electric recliner that

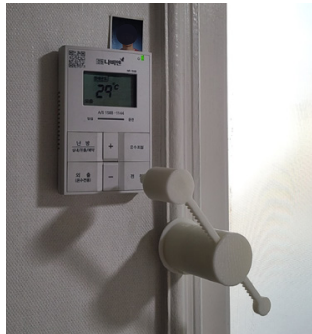

[H2] Thermostat that can be operated outside the home

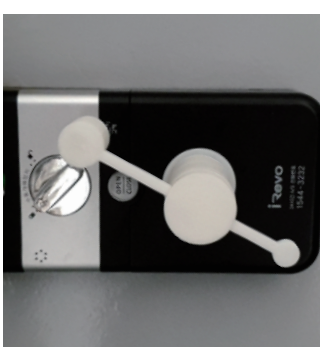

[H4] Door lock that opens when the user comes near the home

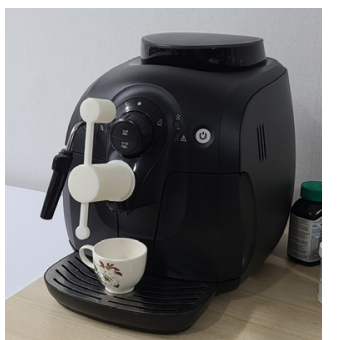

[H7] Coffee machine that operates automatically in the morning

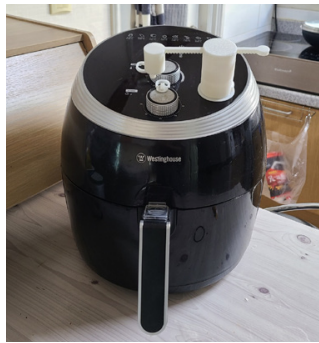

[H3] Air fryer that can be operated with a mobile phone

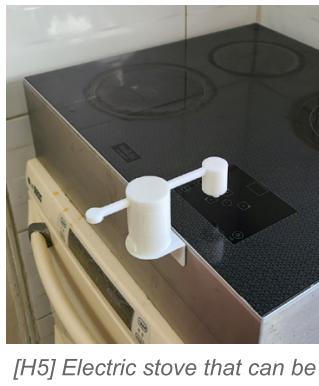

[H5] Electric stove that can be

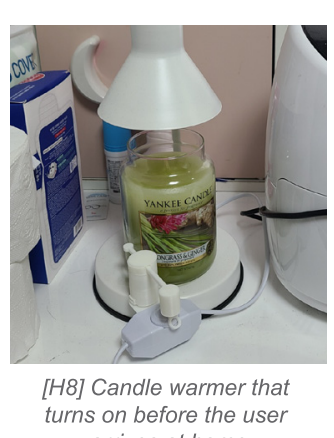

turns on before the that arrives at home

\section{$\checkmark$ par} H3's air conditioner remote control and H5's electric stove can be operated from outside the home. P4B and $\mathrm{P} 6 \mathrm{~A}$ wanted to control the ventilator and radio by voice, respectively. H1's gas valve and H4's door lock are automated products that operate based on the user's condition or behavior, while H5's TV remote control and H7's electric recliner sofa memorize the user's personal settings. Furthermore, P3A wanted the water dispenser to be intelligently automated like an assistant robot when cooking. Participants were also able to easily imagine and plan their desired smart home with the IoTIZER. For example, P2A wanted to set the operating times for various products to keep his own routine, and P7B wanted to set up a control station with several remote controls in one place. While these features are not unique or new, MHD is considered valuable because users can create desired functions when they need, and they can utilze ordinary products. 


\section{DISCUSSION}

\section{Benefits of Versatile MHD}

Overall, the MHD was considered an efficient alternative to providing the core experience of IoT technology. As with previous research [5], most participants were disinclined to invest excessive costs for the convenience of IoT. Therefore, they expected to utilize an available and versatile MHD. This approach is also eco-friendly because it does not discard existing ordinary products. P2A said "If all home appliances around the world are replaced with new ones, there will be terrible amounts of waste. So it is attractive that existing resources can be used."

MHD is sometimes expected to be more scalable than commercial IoT products. The IoTIZER allows highlevel customization via the setting of product operation sequence, time, and conditions. P7B said "Existing IoT can't set scenarios flexible enough as I wanted. For example, in the case of an air conditioner, there is no function to set a specific scenario to operate sequentially." Meanwhile, several participants expected to expand the MHD to include mechanical devices as well as typical interfaces. Assuming the power of the MHD is sufficient, ideas such as turning doorknobs (P8A), opening windows (P7A), and watering plants (P4A) were proposed.

\section{Roles and Values Expected of MHD}

MHD may play an important role in the domestic IoT environment. This is a precious technology these days when IoT replaces existing ordinary products. According to previous user studies on IoT environments experiences, the needs for smart homes are personal and diverse [24, 34]. In addition, smart home devices are not upgraded simultaneously but are introduced gradually [3]. Therefore, users need a way to experience the different roles of existing products and to test new features according to their personal preferences. These opportunities may still be needed even after IoT technology is fully deployed in the domestic environment. Since a smart home needs a lot of iteration in terms of software and hardware until it is customized as desired [5], MHD that can cost-effectively generate an IoT experience could become a great testing tool.

The deployment of IoT products in the home environment will surely provide many benefits to users. But deployment isn't just about providing technology. It is also important to support users can become familiar with the technology and know their needs and preferences. A versatile and easy-to-use MHD can allow users to smoothly transition their existing environment to the IoT environment while maintaining the conventional value.

\section{Future Considerations for Designing MHD}

To design an IoTIZER as a versatile MHD, we focused on two points: (1) a mechanism to manipulate various types of physical interfaces and (2) strategies to install the device for diverse product shapes. The IoTIZER was evaluated positively in terms of versatility in physical manipulation. However, there were limitations as well. In this section, we describe future considerations for providing a satisfactory MHD experience in the real world based on the limitations of IoTIZER found in the study.

First, stability and usability must be ensured. This requires a robust installation method, an error-free operation method, and durability for long-term use. The difficulty of the initial setup process must also be reduced. For example, the setup process could be automated by establishing the pipeline as Facade [13] or via machine learning-based computer vision technology. Second, the installation of an MHD should not interfere with the existing experience. For example, when the user directly manipulates the product, the MHD should not be physically obstructed or restrict movement. The MHD also shouldn't obscure the display or text information. Thus, its design should allow the user to freely select the installation location. Third, the software system must allow users to customize the MHD freely. Currently, the IoTIZER supports the selection of the mechanism type and the manipulation sequence. For users to implement various scenarios, however, more highlevel authoring must be enabled, such as time intervals when manipulating the interface. The software must connect with an existing IoT infrastructure to support users' implementation of desired plans. In particular, rule settings using voice agents or various sensors can be considered. Finally, an MHD should be aesthetically designed for diverse products and environments. Because such a design is challenging, some alternatives can be considered to help create custom devices by diversifying colors, providing additional accessories, or creating intimacy by using famous characters.

One potential solution to these challenges is a mechanical hijacking robot rather than a device. Recent technological developments are introducing personal service robots into the home. If these robots are commercialized before the full deployment of the IoT, they could more intelligently provide the MHD experience.

\section{CONCLUSION}

In this pictorial, we introduced the IoTIZER, a versatile MHD that creates an IooT (Internet of Old Things). We described the design process, key features, usage scenario, and implementation details of IoTIZER We also shared lessons from a user study that took place in users' homes. The potential of IoTIZER were appreciated as a versatile MHD that could provide new value to individuals' IoT experience based on several advantages. We highlighted improvement areas of the IoTIZER in terms of hardware, software, and integration with other interaction technologies. We hope that our work will inspire researchers and designers to envision a better IoT environment where past conventions are preserved. 


\section{ACKNOWLEDGMENT}

This research was supported by the 4th BK21 through the National Research Foundation of Korea(NRF) funded by the Ministry of Education(MOE)((NO.4120200913638), and the KAIST(Attachable physical IoT controller transforming legacy products to smart IoT products, Venture Research Program for Master's and PhD Students in the College of Engineering).

\section{REFERENCES}

[1] Raef Abdallah, Lanyu Xu, and Weisong Shi 2017. Lessons and experiences of a DIY smart home. In Proceedings of the Workshop on Smart Internet of Things. 1-6.

[2] Ambe, A. H., Brereton, M., Soro, A., Chai, M. Z., Buys, L., \& Roe, P. (2019, May). Older people inventing their personal internet of things with the IoT un-kit experience. In Proceedings of the 2019 CHI Conference on Human Factors in Computing Systems (pp. 1-15).

[3] Jordan Ash, Monica Babes, Gal Cohen, Sameen Jalal, Sam Lichtenberg, Michael Littman, Vukosi Marivate, Phillip Quiza, Blase Ur, and Emily Zhang. 2011. Scratchable devices: user-friendly programming for household appliances. In International Conference on Human-Computer Interaction. Springer, 137-146.

[4] Berger, A., Ambe, A. H., Soro, A., De Roeck, D., \& Brereton, M. (2019, June). The stories people tell about the home through IoT toolkits. In Proceedings of the 2019 on Designing Interactive Systems Conference (pp. 7-19).
[5] AJ Bernheim Brush, Bongshin Lee, Ratul Mahajan, Sharad Agarwal, Stefan Saroiu, and Colin Dixon. 2011. Home automation in the wild: challenges and opportunities. In proceedings of the SIGCHI Conference on Human Factors in Computing Systems. 2115-2124.

[6] Stuart K Card, Jock D Mackinlay, and George G Robertson. 1990. The design space of input devices. In Proceedings of the SIGCHI conference on Human factors in computing systems. 117-124.

[7] Marshini Chetty, Ja-Young Sung, and Rebecca E Grinter. 2007. How smart homes learn: The evolution of the networked home and household. In International Conference on Ubiquitous Computing. Springer, 127-144.

[8] Joëlle Coutaz and James L Crowley. 2016. A firstperson experience with end-user development for smart homes. IEEE Pervasive Computing 15, 2 (2016), 26-39.

[9] Rémy Dautriche, Camille Lenoir, Alexandre Demeure, Cédric Gérard, Joëlle Coutaz, and Patrick Reignier. 2013. End-user-development for smart homes: relevance and challenges.

[10] Scott Davidoff, Nicolas Villar, Alex S. Taylor, and Shahram Izadi. 2011. Mechanical Hijacking: How Robots Can Accelerate UbiComp Deployments. In Proceedings of the 13th International Conference on Ubiquitous Computing (Beijing, China) (UbiComp '11). ACM, New York, NY, USA, 267-270. https://doi. org/10.1145/2030112.2030148

[11] Jared Alan Frank and Vikram Kapila. 2015. Path bending: Interactive human-robot interfaces with collision-free correction of user-drawn paths. In Proceedings of the 20th International Conference on Intelligent User Interfaces. 186-190.
[12] FROLIC studio. 2018. Smartians. Retrieved February 10, 2021 from https://www.frolicstudio. com/portfolio/smartians.

[13] Anhong Guo, Jeeeun Kim, Xiang'Anthony' Chen, Tom Yeh, Scott E Hudson, Jennifer Mankoff, and Jeffrey P Bigham. 2017. Facade: Auto-generating tactile interfaces to appliances. In Proceedings of the 2017 CHI Conference on Human Factors in Computing Systems. 5826-5838.

[14] Justin Huang and Maya Cakmak. 2015. Supporting mental model accuracy in trigger-action programming. In Proceedings of the 2015 ACM International Joint Conference on Pervasive and Ubiquitous Computing. 215-225.

[15] Hejhome. 2019. IoT Smart Remote Control Hub. Retrieved February 10, 2021 from https:/www. hej.life/shop_view/?idx=8/.

[16] Hilary Hutchinson, Wendy Mackay, Bo Westerlund, Benjamin B Bederson, Allison Druin, Catherine Plaisant, Michel Beaudouin-Lafon, Stéphane Conversy, Helen Evans, Heiko Hansen, et al. 2003. Technology probes: inspiring design for and with families. In Proceedings of the SIGCHI conference on Human factors in computing systems. 17-24.

[17] IFTTT. Retrieved February 10, 2021 from https:// ifttt.com/.

[18] I/O Inc. 2016. Switcher. Retrieved February 10, 2021 from https://try.i-o.studio/\#/switcher.

[19] Jiahao Li, Jeeeun Kim, and Xiang'Anthony' Chen. 2019. Robiot: A Design Tool for Actuating Everyday Objects with Automatically Generated 3D Printable Mechanisms. In Proceedings of the 32nd Annual ACM Symposium on User Interface Software and Technology. 673-685. 
[20] Kexi Liu, Daisuke Sakamoto, Masahiko Inami, and Takeo Igarashi. 2011. Roboshop: multilayered sketching interface for robot housework assignment and management. In Proceedings of the SIGCHI Conference on Human Factors in Computing Systems. 647-656.

[21] Luxrobo. 2018. MODI. Retrieved February 10, 2021 from http://modi.luxrobo.com/.

[22] Teyssier, M., Bailly, G., Pelachaud, C., \& Lecolinet, E. (2018, October). MobiLimb: Augmenting Mobile Devices with a Robotic Limb. In Proceedings of the 31st Annual ACM Symposium on User Interface Software and Technology (pp. 53-63).

[23] Sarah Mennicken and Elaine M Huang. 2012. Hacking the natural habitat: an in-the-wild study of smart homes, their development, and the people who live in them. In International conference on pervasive computing. Springer, 143-160.

[24] Sarah Mennicken, Jo Vermeulen, and Elaine M Huang. 2014. From today's augmented houses to tomorrow's smart homes: new directions for home automation research. In Proceedings of the 2014 ACM International Joint Conference on Pervasive and Ubiquitous Computing. 105-115.

[25] Naran Inc. 2018. Microbot Push. Retrieved February 10, 2021 from https://microbot.is/products/ microbot-push

[26] Mark W Newman, Ame Elliott, and Trevor F Smith. 2008. Providing an integrated user experience of networked media, devices, and services through end-user composition. In International Conference on Pervasive Computing. Springer, 213-227.

[27] John F Pane, Brad A Myers, et al. 2001. Studying the language and structure in non-programmers' solutions to programming problems. International Journal of Human-Computer Studies 54, 2 (2001), 237-264
[28] Raf Ramakers, Fraser Anderson, Tovi Grossman, and George Fitzmaurice. 2016. RetroFab: A Design Tool for Retrofitting Physical Interfaces Using Actuators, Sensors and 3D Printing. In Proceedings of the 2016 CHI Conference on Human Factors in Computing Systems (San Jose, California, USA) (CHI '16). ACM, New York, NY, USA, 409-419. https://doi.org/10.1145/2858036.2858485

[29] SAM Labs. 2014. STEAM Course Kit. Retrieved February 10, 2021 from https://int.samlabs.com/.

[30] Gary Scott and Jeannette Chin. 2013. A DIY approach to pervasive computing for the Internet of Things: A smart alarm clock. In 2013 5th Computer Science and Electronic Engineering Conference (CEEC). IEEE, 57-60.

[31] Shirafuji, S., Ikemoto, S., \& Hosoda, K. (2014). Development of a tendon-driven robotic finger for an anthropomorphic robotic hand. The International Journal of Robotics Research, 33(5), 677-693.

[32] Leila Takayama, Caroline Pantofaru, David Robson, Bianca Soto, and Michael Barry. 2012. Making technology homey: finding sources of satisfaction and meaning in home automation. In Proceedings of the 2012 ACM conference on ubiquitous computing. 511-520.

[33] The Chamberlain Group, Inc. 2020. Liftmaster. Retrieved August 30, 2020 from https://https://www. myq.com/smart-lock/.

[34] Blase Ur, Elyse McManus, Melwyn Pak Yong Ho, and Michael L Littman. 2014. Practical triggeraction programming in the smart home. In Proceedings of the SIGCHI Conference on Human Factors in Computing Systems. 803-812.

[35] Wonderlabs Inc. 2018. SwitchBot. Retrieved February 10, 2021 from https://www.switch-bot.com/ bot.
[36] Jong-bum Woo and Youn-kyung Lim. 2015. User experience in do-it-yourself-style smart homes. In Proceedings of the 2015 ACM international jointconference on pervasive and ubiquitous computing. 779-790.

[37] Xu, Z., Kumar, V., Matsuoka, Y., \& Todorov, E. (2012, June). Design of an anthropomorphic robotic finger system with biomimetic artificial joints. In 2012 4th IEEE RAS \& EMBS International Conference on Biomedical Robotics and Biomechatronics (BioRob) (pp. 568-574). IEEE. 